\title{
Association of serum methionine metabolites with non-alcoholic fatty liver disease: a cross-sectional study
}

\section{Yi Tang}

Sun Yat-Sen University School of Public Health

\section{Xu Chen}

Sun Yat-Sen University School of Public Health

\section{Qian Chen}

Sun Yat-Sen Memorial Hospital

\section{Jinghe Xiao}

Sun Yat-Sen University School of Public Health

Jiaxin Mi

Sun Yat-Sen University School of Public Health

Qiannan Liu

Sun Yat-Sen University School of Public Health

\section{Yiran You}

Sun Yat-Sen University School of Public Health

\section{Yuming Chen}

Sun Yat-Sen University School of Public Health

Wenhua Ling ( $\square$ lingwh@mail.sysu.edu.cn )

Sun Yat-Sen University https://orcid.org/0000-0002-5468-6605

\section{Research Article}

Keywords: Methionine metabolites, Non-alcoholic fatty liver disease, Hepatic steatosis, Sadenosylmethionine, S-adenosylhomocysteine, homocysteine

Posted Date: November 9th, 2021

DOI: https://doi.org/10.21203/rs.3.rs-1046119/v1

License: (c) (1) This work is licensed under a Creative Commons Attribution 4.0 International License. Read Full License 
Version of Record: A version of this preprint was published at Nutrition \&amp; Metabolism on March 18th, 2022. See the published version at https://doi.org/10.1186/s12986-022-00647-7. 


\section{Abstract \\ Background}

Non-alcoholic fatty liver disease (NAFLD) is viewed as the hepatic manifestation of metabolic syndrome. Methionine metabolites have been linked to metabolic syndrome and its related diseases. Whether methionine metabolites levels are associated with NAFLD remains unclear. The study aimed to assess the association between methionine metabolites and NAFLD.

\section{Methods}

This cross-sectional study included a total of 2814 individuals aged $40-75$ years old. All participants underwent anthropometric measurements, laboratory tests and abdominal ultrasonography. Multivariable logistic regression analysis was performed to estimate the association of methionine metabolites with NAFLD.

\section{Results}

Overall, 1446 with and 1368 without NAFLD were enrolled in this study. Participants with NAFLD had significantly higher serum S-adenosylmethionine (SAM), S-adenosylhomocysteine (SAH) and homocysteine (Hcy) levels, and a lower S-adenosylmethionine/S-adenosylhomocysteine (SAM/SAH) ratio than those without NAFLD (all $P<0.001$ ). After adjusting multiple confounders, odds ratios (ORs) (95\% confidence interval [CI]) for quartile 4 vs quartile 1 of SAH, Hcy and SAM/SAH ratio were 1.65 (1.27$2.14), 1.63(1.26-2.12)$ and $0.63(0.49-0.83)$, respectively (all $P$ for trend $<0.01)$. In addition, serum $S A H$, Hcy levels and SAM/SAH ratio were significantly correlated with degree of hepatic steatosis (all $P$ for trend $<0.001$ ).

\section{Conclusion}

Elevated serum SAH, Hcy levels and lower SAM/SAH ratio were independently associated with the presence of NAFLD in middle-aged and elder Chinese.

\section{Introduction}

Non-alcoholic fatty liver disease (NAFLD) has been emerging as the leading chronic liver disease and a significant global health burden gradually, affecting up to $25 \%$ of the world population ${ }^{[1]}$. In China, the prevalence of NAFLD reached $32.9 \%$ in 2018 . And the incidence is substantially rising over past decades, by $4.6 \%$ in $2011-2013$ to $5.2 \%$ in $2014-2016^{[2]}$. NAFLD, ranging from isolated hepatic steatosis to nonalcoholic steatohepatitis (NASH) and cirrhosis ${ }^{[3]}$, is viewed as the hepatic manifestation of metabolic 
syndrome ${ }^{[4]}$. The progression of NAFLD is often unpredictable and asymptomatic, which makes it easy to be ignored ${ }^{[5]}$. Therefore, it is necessary to identify new biomarkers in term of prediction of occurrence and development of NAFLD.

Methionine metabolism is a key component of one-carbon metabolism which plays an important role in a broad range of metabolic diseases ${ }^{[6]}$. At this aspect, several intermediate metabolites, such as Sadenosylmethionine (SAM), S-adeosyl-homocysteine (SAH) and homocysteine (Hcy), have been received great attention (Their relationships were shown in Fig. S1). As the direct metabolite of methionine, SAM is the universal methyl donor for cellular methylation. SAH as a major byproduct of methylation, is the potent feedback inhibitor of SAM-dependent methyltransferases ${ }^{[7]}$. Elevated plasma SAH concentrations were associated with an increased risk of cardiovascular events in coronary angiography patients ${ }^{[8]}$. Moreover, S-adenosylmethionine/S-adenosylhomocysteine (SAM/SAH) ratio is considered as the methylation potential or capacity index. A low ratio was associated with increased risks of metabolic diseases such as chronic kidney disease and cardiovascular disease ${ }^{[9,10]}$. Hcy is produced from SAH by reversible reaction of SAH hydrolase $(\mathrm{SAHH})$, and thus is intrinsically related to cellular methylation status as well ${ }^{[11]}$. Hyperhomocysteinemia may be implicated in the development of many metabolic diseases, such as obesity and type 2 diabetes, and a recent study indicated that high plasm Hcy levels could aggravate insulin resistance and vascular endothelial dysfunction in patients with type 2 diabetes $^{[12]}$. Studies have shown that methionine metabolism is highly active in the hepatocytes and serum methionine metabolites levels were correlated with hepatic methionine metabolism activity. Thus, serum methionine metabolites might be the indicators of liver methionine metabolism ${ }^{[13]}$.

The hallmark of NAFLD is the abnormal accumulation of lipid in the liver ${ }^{[14]}$. As the major site of both methionine and lipid metabolism, recently, emerging evidence indicates that methionine metabolites, including SAM, SAH and Hcy, are critical determinants of hepatic lipid levels ${ }^{[15]}$. Animal experiments showed that methionine metabolism dysregulation might lead to decreased transport of lipids through hypomethylation of phosphatidylcholine (PC) and increased synthesis of lipids via feedbacking activated sterol regulatory element-binding proteins $\left(\right.$ SREBPs ${ }^{[16,17]}$. Besides, hepatic global DNA hypomethylation was associated with NAFLD, hepatic inflammation and fibrosis ${ }^{[18]}$, and methyl donor supplementation prevented the progression of NAFLD ${ }^{[15]}$. Although it has been well documented in animal models, there are limited data regarding the association of serum methionine metabolites with the presence and severity of NAFLD in the population. Humans with SAHH deficiency, a rare genetic disease charactered by sharply raising SAH levels, possessed mildly active chronic hepatitis with moderate portal fibrosis ${ }^{[19]}$. At present, although a few epidemiology studies investigated whether methionine metabolites are associated with hepatic metabolic diseases, but results are inconsistent ${ }^{[20,21]}$. Therefore, this study sought to evaluate the association between methionine metabolites and NAFLD through a communitybased population of middle-aged and elderly Chinese.

\section{Materials And Methods}




\subsection{Study design and population}

We conducted a cross-sectional analysis in the Guangzhou Nutrition and Health Study (GNHS), which was performed among the middle-aged and elderly community residents ( $40-75$ years old) in southern China, to evaluate the relationship between the serum methionine metabolites levels and NAFLD.

Exclusion criteria included: excess alcohol consumption ( $\geq 140 \mathrm{~g} /$ week in men, $\geq 70 \mathrm{~g} /$ week in women); viral or autoimmune hepatitis; drug- or toxin-induced liver diseases; biliary obstructive diseases; genetic liver diseases; chronic kidney disease or renal failure; HIV infection; any type of cancer; current treatment with systemic corticosteroids or anti-inflammatory therapy; or pregnancy ${ }^{[22]}$. Finally, 2814 participants were included in the analysis (Fig. 1). The study was approved by the Ethics Committee of the School of Public Health at Sun Yat-sen University and all eligible participants signed informed consent.

\subsection{Clinical and laboratory measurements}

Study participants were invited for a single day visit, in which they underwent comprehensive physical examination, fasting blood test, hepatitis virus test, abdominal B-scan ultrasonography, a face-to-face interview using a structured questionnaire including demographic characteristics, lifestyle, and habits (e.g., health status, alcohol consumption, smoking, and physical activity) and history of chronic diseases.

Height and weight were obtained with participants wearing light clothes and no shoes. The body mass index (BMI) was calculated as weight in kilograms divided by the square of height in meters $\left(\mathrm{kg} / \mathrm{m}^{2}\right)$. The waist-to-hip ratio (WHR) was calculated as waist circumference $(\mathrm{cm})$ divided by hip circumference $(\mathrm{cm})$. The metabolic equivalent (MET) intensity was calculated to estimate daily physical activity using a 24-h physical activity questionnaire. The fat mass of the trunk region was quantified using dual-energy X-ray absorptiometry scans (Discovery W; Hologic Inc., Waltham, MA, USA). Venous blood samples were collected from all the participants in the morning after an overnight fast, and serum was separated and immediately stored within $2 \mathrm{~h}$ of collection at $-80^{\circ} \mathrm{C}$ until analysis. The samples used for the analysis of biochemical values, including serum fasting glucose, fasting insulin, triglycerides (TG), total cholesterol (TC), high-density lipoprotein cholesterol (HDL-C), low-density lipoprotein cholesterol (LDL-C), aspartate aminotransferase (AST), alanine transaminase (ALT), alkaline phosphatase (ALP), uric acid (UA), and high-sensitivity C-reactive protein (hsCRP). All values were measured by a Hitachi 7600-010 automated analyzer (Hitachi, Tokyo, Japan). Insulin resistance (IR) was evaluated by the homeostasis model assessment $(\mathrm{HOMA})$ calculated as fasting glucose $(\mathrm{mM}) \times$ fasting insulin $(\mathrm{mU} / \mathrm{L}) / 22.5$.

Serum methionine metabolites were measured by ultra-high performance liquid chromatography coupled with tandem mass spectrometry (UHPLC/MS-MS, Agilent Technologies, Inc., Santa Clara, CA, USA), as described previously with some differences ${ }^{[23,24]}$. Serum samples $(50 \mu \mathrm{L})$ diluted in ultrapure water $(4: 1$, v/v) were incubated in $37^{\circ} \mathrm{C}$ for $15 \mathrm{~min}$ after spiking $10 \mu \mathrm{L}$ DL-dithiothreitol (DTT, $50 \mathrm{mM}$ ) and $10 \mu \mathrm{L}$ mixture of deuterium-labeled internal standards $\left({ }^{2} \mathrm{H}_{3}-\mathrm{SAM}, 500 \mathrm{nM} ;{ }^{2} \mathrm{H}_{4}-\mathrm{SAH}, 500 \mathrm{nM} ;{ }^{2} \mathrm{H}_{4}-\mathrm{Hcy}, 5 \mu \mathrm{M}\right)$. Subsequently, $30 \mu \mathrm{L}$ perchloric acid $(1 \mathrm{M})$ was added to samples for protein precipitation. Then, the samples were centrifuged at $15,000 \times \mathrm{g}$ for $10 \mathrm{~min}$ at $4^{\circ} \mathrm{C}$. Finally, the supernatants were filtered by a 0.22 
$\mu \mathrm{m}$ membrane. The methionine metabolites were separated through an Acquity BEH C18 column ( $2.1 \times$ $50 \mathrm{~mm}$; i.d. $1.7 \mu \mathrm{m}$ ) (Waters Corp., Milford, MA, USA), detected by Agilent 1290 Infinity II UHPLC system coupled with Agilent 6410 Triple Quadrupole LC/MS system, and quantified in multiple reaction monitoring mode. The linearity regression coefficients of SAM, SAH and Hcy were more than 0.99 , with inter- and intra-assay coefficients of variation less than $10 \%$ simultaneously (Table S1).

\subsection{Ultrasonography examination}

The abdominal ultrasonic examination was performed by the same group of trained and experienced ultrasonographists who were blind to the clinical and laboratory data, using a Doppler sonography (Sonoscape SSI-5500, Shenzhen, China) equipped with a 3.5-MHz probe. Hepatic steatosis was diagnosed by abdominal characteristic echo patterns according to standardized criteria issued by the Chinese Liver Disease Association. NAFLD was defined as diffuse fatty liver following exclusion of alcohol consumption, viral, or autoimmune liver diseases. Moreover, according to the echogenicity, the severity of hepatic steatosis was graded as follows: absent, normal echo pattern; mild, slight and diffuse increase in fine echoes in liver parenchyma with normal visualization of intrahepatic vessel borders and diaphragm; moderate, middling and diffuse enhancement in fine echoes with marginally impaired visualization of intrahepatic vessels and diaphragm; severe, marked elevation in fine echoes with poor or non-visualization of the intrahepatic vessel borders, diaphragm, and posterior right lobe of the liver ${ }^{[25]}$.

There was a good consistency for ultrasound examination against abdominal computed tomography (Spearman $\mathrm{r}=0.905, \mathrm{~K}=0.691$, and total agreement $=85 \%, P<.001$ ) and a good reliability among operators (Spearman $\mathrm{r}=0.911, \mathrm{~K}=0.875$, and total agreement $=93 \%, P<0.001$ ).

\subsection{Statistical analysis}

We computed descriptive statistics of baseline characteristics and serum methionine metabolites concentrations, presented as mean \pm standard deviation (SD), median [25th, 75th percentiles], or frequencies with percentages, as appropriate. We evaluated differences in study variables between NAFLD and non-NAFLD participants by the unpaired Student's $t$ test or Mann-Whitney $U$ test for numerical variables and the Chi-Square test or Fisher's exact test for categorical data. The correlations between serum methionine metabolites levels and relevant metabolic factors were examined by Spearman correlation coefficients. Multivariable logistic regression was performed to analyze the association between methionine metabolites and NAFLD. All participants were classified into quartiles by SAM, SAH, Hcy levels and SAM/SAH ratio. Both univariable and multivariable $P$ for trend between quartiles of methionine metabolites and prevalence of NAFLD were calculated by logistic regression analysis. Covariates in the multivariable model, which were chosen according to their clinical importance as well as statistical significance, included age, gender, BMI, WHR, trunk fat ratio, physical activity, current smoking, current drinking, history of hypertension, diabetes, dyslipidemia and heart disease, HOMA-IR, TC, TG, HDL, LDL, AST/ALT ratio, UA, ALP and hsCRP. In order to test whether the results were consistent among different subgroups, we stratified study participants by gender (female vs. male), age ( $<65 \mathrm{vs.} \geq 65$, years), $\mathrm{BMI}\left(<24 \mathrm{vs} . \geq 24, \mathrm{~kg} / \mathrm{m}^{2}\right), \operatorname{HOMA}-\mathrm{IR}(<2.5 \mathrm{vs} . \geq 2.5)$, and TG ( $<1.7 \mathrm{vs} . \geq 1.7 \mathrm{mM}$ ) levels and 
performed logistics regression analysis after adjusting for the same confounding factors. Moreover, we performed univariate and multivariate comparisons of methionine metabolites levels across hepatic steatosis groups by Kruskal-Wallis one-way analysis of variance (ANOVA) for $k$ samples and analysis of covariate (ANCOVA), respectively. Data manipulation and statistical analyses were performed using SPSS version 25 software (IBM Inc., Chicago, IL). $P<0.05$ (two-tailed test) was considered statistically significant.

\section{Results}

\subsection{Characteristics of the study population}

There were eligible 1446 (51.39\%) NAFLD and 1368 (48.61\%) non-NAFLD participants in the present study. The mean age of the study population was $60.84 \pm 5.76$ years, and 868 (30.85\%) participants were male. The baseline characteristics of participants with and without NAFLD are shown (Table 1). NAFLD participants were much more likely to have higher BMI, WHR, HOMA-IR, TG, higher prevalence rates of hypertension and diabetes and dyslipidemia, and less likely to have higher physical activities, HDL-C and AST/ALT ratio than those without NAFLD. However, there were no differences in LDL-C and TC.

Participants with NAFLD had higher concentrations of serum SAM (91.06 vs $85.67 \mathrm{nM}$ ), SAH (17.74 vs $14.50 \mathrm{nM})$ and Hcy (13.61 vs $12.51 \mu \mathrm{M})$, and lower SAM/SAH ratio (5.31 vs 6.09) than those without NAFLD (all $P<0.001)$. 
Table 1

Baseline characteristics of populations with and without NAFLD.

\begin{tabular}{|c|c|c|c|c|}
\hline Characteristics & Overall & NAFLD $(n=1446)$ & $\begin{array}{l}\text { Non-NAFLD } \\
(n=1368)\end{array}$ & $\begin{array}{l}P \\
\text { value }\end{array}$ \\
\hline \multicolumn{5}{|l|}{ Demographics } \\
\hline Age (years) & $60.84 \pm 5.76$ & $61.97 \pm 5.45$ & $60.71 \pm 6.06$ & 0.221 \\
\hline Sex (male) & $868(30.85)$ & $468(32.37)$ & $400(29.24)$ & 0.073 \\
\hline \multicolumn{5}{|l|}{ Anthropometrics } \\
\hline $\mathrm{BMI}\left(\mathrm{kg} / \mathrm{m}^{2}\right)$ & $23.61 \pm 3.16$ & $25.01 \pm 3.00$ & $22.13 \pm 2.60$ & $\begin{array}{l}< \\
0.001\end{array}$ \\
\hline Waist (cm) & $84.91 \pm 8.80$ & $88.34 \pm 8.15$ & $81.29 \pm 7.98$ & $\begin{array}{l}<.001 \\
0.001\end{array}$ \\
\hline WHR & $0.92 \pm 0.07$ & $0.94 \pm 0.06$ & $0.90 \pm 0.07$ & $\begin{array}{l}<.001 \\
0.00\end{array}$ \\
\hline Trunk fat percentage (\%) & $33.62 \pm 6.74$ & $35.65 \pm 5.85$ & $31.49 \pm 6.95$ & $\begin{array}{l}<.001 \\
0.001\end{array}$ \\
\hline $\mathrm{SBP}(\mathrm{mmHg})$ & $125.21 \pm 17.93$ & $128.37 \pm 17.51$ & $121.88 \pm 17.76$ & $\begin{array}{l}< \\
0.001\end{array}$ \\
\hline $\mathrm{DBP}(\mathrm{mmHg})$ & $75.57 \pm 10.37$ & $77.56 \pm 10.16$ & $73.48 \pm 10.18$ & $\begin{array}{l}<.001 \\
0.001\end{array}$ \\
\hline \multicolumn{5}{|l|}{ Blood biochemical indices } \\
\hline Fasting glucose (mM) & $4.80[4.39,5.31]$ & $4.85[4.43,5.41]$ & $4.72[4.33,5.21]$ & $\dot{0.001}$ \\
\hline Fasting insulin $(\mu \mathrm{U} / \mathrm{mL})$ & $7.77[5.39,11.21]$ & $9.97[7.00,13.86]$ & $6.08[4.42,8.27]$ & $\begin{array}{l}<.001 \\
0.001\end{array}$ \\
\hline HOMA-IR & $1.67[1.11,2.54]$ & $2.18[1.49,3.18]$ & $1.30[0.90,1.84]$ & $\dot{0.001}$ \\
\hline AST/ALT & $1.20[0.96,1.45]$ & $1.09[0.87,1.33]$ & $1.30[1.08,1.56]$ & $\begin{array}{l}< \\
0.001\end{array}$ \\
\hline
\end{tabular}

Values are shown as mean \pm standard deviation, median [25th, 75th percentiles] or frequencies (\%).

Statistical analysis was performed using $t$ test, Mann-Whitney $\mathrm{U}$ test, chi-square test.

NAFLD, non-alcoholic fatty liver disease; BMI, body mass index; WHR, waist-to-hip ratio; SBP, systolic blood pressure; DBP, diastolic blood pressure; HOMA-IR, homoeostasis model assessment of insulin resistance; AST, aspartate aminotransferase; ALT, alanine aminotransferase; TC, total cholesterol; TG, triglycerides; HDL-C, high-density lipoprotein cholesterol; LDL-C, low-density lipoprotein cholesterol; UA, uric acid; ALP, Alkaline phosphatase; hsCRP, high-sensitivity C-reactive protein; SAM, Sadenosylmethionine; SAH, S-adenosylhomocysteine; Hcy, homocysteine; MET, metabolic equivalent of task. 


\begin{tabular}{|c|c|c|c|c|}
\hline Characteristics & Overall & NAFLD $(n=1446)$ & $\begin{array}{l}\text { Non-NAFLD } \\
(n=1368)\end{array}$ & $\begin{array}{l}P \\
\text { value }\end{array}$ \\
\hline $\mathrm{TC}(\mathrm{mM})$ & $5.59 \pm 1.05$ & $5.56 \pm 1.03$ & $5.63 \pm 1.07$ & 0.059 \\
\hline TG (mM) & $1.26[0.90,1.78]$ & $1.44[1.04,2.06]$ & $1.08[0.81,1.50]$ & $\begin{array}{l}< \\
0.001\end{array}$ \\
\hline HDL-C (mM) & $1.43 \pm 0.41$ & $1.32 \pm 0.36$ & $1.55 \pm 0.43$ & $<0.001$ \\
\hline LDL-C (mM) & $3.60 \pm 0.91$ & $3.62 \pm 0.90$ & $3.57 \pm 0.91$ & 0.140 \\
\hline $\mathrm{UA}(\mu \mathrm{M})$ & $350.50 \pm 84.32$ & $364.68 \pm 83.31$ & $335.49 \pm 82.80$ & $\begin{array}{l}< \\
0.001\end{array}$ \\
\hline ALP (U/L) & $\begin{array}{l}70.07[58.87, \\
83.07]\end{array}$ & $\begin{array}{l}71.25[59.47 \\
84.08]\end{array}$ & $\begin{array}{l}68.85[58.23 \\
81.76]\end{array}$ & $\begin{array}{l}< \\
0.001\end{array}$ \\
\hline hsCRP (mg/L) & $0.96[0.57,1.94]$ & $1.19[0.67,2.44]$ & $0.77[0.48,1.38]$ & $<.001$ \\
\hline \multicolumn{5}{|l|}{$\begin{array}{l}\text { Serum methionine } \\
\text { metabolites }\end{array}$} \\
\hline SAM (nM) & $\begin{array}{l}88.07[75.04, \\
110.04]\end{array}$ & $\begin{array}{l}91.06 \text { [76.90, } \\
111.19]\end{array}$ & $\begin{array}{l}85.67[73.62, \\
108.74]\end{array}$ & $<.001$ \\
\hline SAH (nM) & $\begin{array}{l}15.70[11.46 \\
24.55]\end{array}$ & $\begin{array}{l}17.74[12.09 \\
26.24]\end{array}$ & $\begin{array}{l}14.50[10.84 \\
22.64]\end{array}$ & $\begin{array}{l}< \\
0.001\end{array}$ \\
\hline Hcy $(\mu \mathrm{M})$ & $\begin{array}{l}13.06[11.33, \\
16.39]\end{array}$ & $\begin{array}{l}\text { 13.61 [11.66, } \\
17.43]\end{array}$ & $\begin{array}{l}12.51[10.97 \\
15.35]\end{array}$ & $<0.001$ \\
\hline SAM/SAH & $5.68[4.18,7.20]$ & $5.31[3.99,7.01]$ & $6.09[4.47,7.42]$ & $<.001$ \\
\hline \multicolumn{5}{|l|}{ Lifestyle } \\
\hline $\begin{array}{l}\text { Physical activities } \\
\text { (MET/day) }\end{array}$ & $34.05 \pm 5.65$ & $33.69 \pm 5.55$ & $34.43 \pm 5.73$ & $<0.001$ \\
\hline Current smoking & $313(11.12)$ & $164(11.34)$ & $149(10.89)$ & 0.704 \\
\hline Current drinking & $204(7.25)$ & 109 (7.54) & $95(6.94)$ & 0.540 \\
\hline
\end{tabular}

Values are shown as mean \pm standard deviation, median [25th, 75th percentiles] or frequencies (\%).

Statistical analysis was performed using $t$ test, Mann-Whitney $U$ test, chi-square test.

NAFLD, non-alcoholic fatty liver disease; BMI, body mass index; WHR, waist-to-hip ratio; SBP, systolic blood pressure; DBP, diastolic blood pressure; HOMA-IR, homoeostasis model assessment of insulin resistance; AST, aspartate aminotransferase; ALT, alanine aminotransferase; TC, total cholesterol; TG, triglycerides; HDL-C, high-density lipoprotein cholesterol; LDL-C, low-density lipoprotein cholesterol; UA, uric acid; ALP, Alkaline phosphatase; hsCRP, high-sensitivity C-reactive protein; SAM, Sadenosylmethionine; SAH, S-adenosylhomocysteine; Hcy, homocysteine; MET, metabolic equivalent of task. 


\begin{tabular}{|c|c|c|c|c|}
\hline Characteristics & Overall & NAFLD $(n=1446)$ & $\begin{array}{l}\text { Non-NAFLD } \\
(n=1368)\end{array}$ & $\begin{array}{l}P \\
\text { value }\end{array}$ \\
\hline \multicolumn{5}{|c|}{ History of disease } \\
\hline Hypertension & $825(29.35)$ & $518(35.85)$ & $307(22.47)$ & $\begin{array}{l}< \\
0.001\end{array}$ \\
\hline Diabetes & $213(7.58)$ & $127(8.79)$ & $86(6.30)$ & 0.019 \\
\hline Dyslipidemia & 1099 (39.05) & $647(44.74)$ & $452(33.04)$ & $<.001$ \\
\hline Heart diseases & $559(19.91)$ & $302(20.91)$ & $257(18.84)$ & 0.330 \\
\hline \multicolumn{5}{|c|}{ Values are shown as mean \pm standard deviation, median [25th, 75th percentiles] or frequencies (\%). } \\
\hline \multicolumn{5}{|c|}{ Statistical analysis was performed using $t$ test, Mann-Whitney $\mathrm{U}$ test, chi-square test. } \\
\hline \multicolumn{5}{|c|}{$\begin{array}{l}\text { NAFLD, non-alcoholic fatty liver disease; BMI, body mass index; WHR, waist-to-hip ratio; SBP, systolic } \\
\text { blood pressure; DBP, diastolic blood pressure; HOMA-IR, homoeostasis model assessment of insulin } \\
\text { resistance; AST, aspartate aminotransferase; ALT, alanine aminotransferase; TC, total cholesterol; TG, } \\
\text { triglycerides; HDL-C, high-density lipoprotein cholesterol; LDL-C, low-density lipoprotein cholesterol; } \\
\text { UA, uric acid; ALP, Alkaline phosphatase; hsCRP, high-sensitivity C-reactive protein; SAM, S- } \\
\text { adenosylmethionine; SAH, S-adenosylhomocysteine; Hcy, homocysteine; MET, metabolic equivalent of } \\
\text { task. }\end{array}$} \\
\hline
\end{tabular}

The correlations between methionine metabolites and metabolic related factors were shown in Table 2. Among all participants, the significantly positive correlations between SAM, SAH, Hcy and age, BMI, WHR, HOMA-IR, TG, UA and the negative correlations between SAM, SAH, Hcy and HDL-C, AST/ALT ratio were observed. On the contrary, SAM/SAH ratio was inversely correlated with age, BMI, WHR, TG, LDL-C, UA, hsCRP and positively correlated with HDL-C (all $P<0.05)$. 
Table 2

Spearman correlation coefficients of methionine metabolites in cycle and metabolic relevant factors.

\begin{tabular}{|c|c|c|c|c|}
\hline Variables & SAM & SAH & Hcy & SAM/SAH \\
\hline Age (years) & $0.132^{\star \star \star}$ & $0.164^{\star \star \star}$ & $0.159^{\star \star \star}$ & $-0.108^{\star \star \star}$ \\
\hline BMI $\left(\mathrm{kg} / \mathrm{m}^{2}\right)$ & $0.129^{\star \star \star}$ & $0.172^{\star \star \star}$ & $0.170^{\star \star \star}$ & $-0.101^{\star \star \star}$ \\
\hline WHR & $0.179^{* \star *}$ & $0.152^{* \star \star}$ & $0.099^{\star \star \star}$ & $-0.055^{\star \star \star}$ \\
\hline HOMA-IR & $0.126^{\star \star \star}$ & $0.076^{\star \star \star}$ & $0.098^{\star \star \star}$ & 0.017 \\
\hline AST/ALT & $-0.126^{\star \star \star}$ & $-0.087^{\star \star \star}$ & $-0.037^{\star \star \star}$ & 0.020 \\
\hline $\mathrm{TC}(\mathrm{mM})$ & -0.025 & -0.014 & $-0.058^{\star \star}$ & -0.004 \\
\hline TG (mM) & $0.095^{\star \star \star}$ & $0.155^{\star \star \star}$ & $0.087^{\star \star \star}$ & $-0.108^{\star \star \star}$ \\
\hline HDL-C (mM) & $-0.131^{\star \star \star}$ & $-0.163^{\star \star \star}$ & $-0.128^{\star \star \star}$ & $0.092^{\star \star \star}$ \\
\hline LDL-C (mM) & $-0.053^{\star \star}$ & -0.002 & -0.023 & $-0.038^{*}$ \\
\hline $\mathrm{UA}(\mu \mathrm{M})$ & $0.188^{\star \star \star}$ & $0.161^{\star * \star}$ & $0.100^{\star \star \star}$ & $-0.075^{\star \star *}$ \\
\hline ALP (U/L) & -0.001 & 0.011 & 0.001 & -0.014 \\
\hline hsCRP (mg/L) & $0.120^{\star \star \star}$ & $0.130^{\star \star \star}$ & $0.091^{\star * *}$ & $-0.073^{\star \star \star}$ \\
\hline SAM (nM) & - & $0.527^{\star \star \star}$ & $0.193^{\star \star \star}$ & $-0.002^{\star \star \star}$ \\
\hline SAH (nM) & $0.527^{\star \star \star}$ & - & $0.416^{\star \star \star}$ & $-0.815^{\star \star \star}$ \\
\hline Hcy $(\mu \mathrm{M})$ & $0.193^{\star \star \star}$ & $0.416^{\star \star \star}$ & - & $-0.341^{\star \star \star}$ \\
\hline SAM/SAH & $-0.002^{\star \star \star}$ & $-0.815^{\star \star \star}$ & -0.341 & - \\
\hline \multicolumn{5}{|c|}{$\star, P<0.05 ; * \star, P<0.01 ; * \star \star, P<0.001}$. \\
\hline
\end{tabular}

\subsection{Association between methionine metabolites and NAFLD}

Table 3 demonstrated that the proportion of NAFLD tended to increase along with the increased quartiles of SAM, SAH and Hcy levels and the decreased SAM/SAH ratio (all $P$ for trend $<0.001$ ). After adjusting potential confounders, the odds of NAFLD increased with increasing SAH level in all models (all $P$ for trend $<0.001$ ). The adjusted odds ratios (ORs) (95\% confidence interval [CI]) for quartile 4 vs quartile 1 of model 1, model 2 and model 3 were 2.46 (1.97-3.08), 1.74 (1.35-2.24) and 1.65 (1.27-2.14), respectively. In addition, serum Hcy level was also positively associated with NAFLD in all models (all $P$ 
for trend $<0.01)$. The adjusted ORs $(95 \% \mathrm{Cl})$ for quartile 4 vs quartile 1 of model 1 , model 2 and model 3 were 2.41 (1.93-3.01), 1.73 (1.34-2.23) and 1.63 (1.26-2.12), respectively. Meanwhile, the inverse association of SAM/SAH ratio with NAFLD was observed in all models (all $P$ for trend $<0.001$ ). The adjusted ORs $(95 \% \mathrm{Cl})$ in quartile 4 were $0.57(0.46-0.71)$ in model $1,0.62(0.48-0.80)$ in model 2 , and $0.63(0.49-0.83)$ in model 3 . However, there was no statistically significant association between SAM level and the odds of NAFLD. 
Table 3

Adjusted ORs $(95 \% \mathrm{Cl})$ for NAFLD prevalence by quartiles of serum methionine metabolites.

Quartiles of serum $1 \mathrm{C}$ metabolites

$P$ for trend

\section{Quartile 1 Quartile 2 Quartile 3 Quartile 4}

\begin{tabular}{|c|c|c|c|c|c|}
\hline SAM (nM) & $<75.05$ & $75.05-88.06$ & $88.07-110.03$ & $>110.04$ & \\
\hline NAFLD (\%) & 44.52 & 50.64 & 56.45 & 53.91 & $<0.001$ \\
\hline Model 1 & 1.00 & $1.34(1.08,1.66)$ & $1.66(1.33,2.06)$ & $1.43(1.15,1.79)$ & $<0.001$ \\
\hline Model 2 & 1.00 & $1.07(0.83,1.38)$ & $1.32(1.03,1.70)$ & $0.99(0.77,1.28)$ & 0.636 \\
\hline Model 3 & 1.00 & $1.01(0.78,1.31)$ & $1.24(0.96,1.61)$ & $0.97(0.74,1.26)$ & 0.795 \\
\hline SAH (nM) & $<11.46$ & $11.46-15.69$ & $15.70-24.52$ & $>24.53$ & \\
\hline NAFLD (\%) & 40.48 & 49.21 & 51.28 & 64.58 & $<0.001$ \\
\hline Model 1 & 1.00 & $1.38(1.11,1.71)$ & $1.50(1.21,1.87)$ & $2.46(1.97,3.08)$ & $<0.001$ \\
\hline Model 2 & 1.00 & $1.16(0.92,1.51)$ & $1.27(0.99,1.63)$ & $1.74(1.35,2.24)$ & $<0.001$ \\
\hline Model 3 & 1.00 & $1.16(0.90,1.50)$ & $1.27(0.98,1.65)$ & $1.65(1.27,2.14)$ & $<0.001$ \\
\hline Hcy ( $\mu \mathrm{M})$ & $<11.33$ & $11.33-13.05$ & $13.06-16.37$ & $>16.38$ & \\
\hline NAFLD (\%) & 40.83 & 49.79 & 51.07 & 63.87 & $<0.001$ \\
\hline Model 1 & 1.00 & $1.42(1.15,1.76)$ & $1.42(1.14,1.76)$ & $2.41(1.93,3.01)$ & $<0.001$ \\
\hline Model 2 & 1.00 & $1.19(0.93,1.53)$ & $1.07(0.84,1.38)$ & $1.73(1.34,2.23)$ & $<0.001$ \\
\hline Model 3 & 1.00 & $1.23(0.96,1.60)$ & $1.09(0.84,1.41)$ & $1.63(1.26,2.12)$ & 0.001 \\
\hline SAM/SAH & $<4.16$ & $4.16-5.74$ & $5.75-7.21$ & $>7.22$ & \\
\hline NAFLD (\%) & 60.26 & 53.79 & 46.75 & 44.57 & $<0.001$ \\
\hline Model 1 & 1.00 & $0.77(0.62,0.96)$ & $0.60(0.41,0.75)$ & $0.57(0.46,0.71)$ & $<0.001$ \\
\hline Model 2 & 1.00 & $0.81(0.63,1.04)$ & $0.64(0.50,0.82)$ & $0.62(0.48,0.80)$ & $<0.001$ \\
\hline Model 3 & 1.00 & $0.80(0.62,1.03)$ & $0.64(0.49,0.83)$ & $0.63(0.49,0.83)$ & $<0.001$ \\
\hline
\end{tabular}

Model 1: adjusted for age, sex;

model 2: adjusted as for model 1 plus BMI, WHR, trunk fat ratio, physical activity (MET/day), current smoking, current drinking, history of hypertension, diabetes, dyslipidemia and heart disease;

model 3: adjusted as for model 2 plus HOMA-IR, TC, TG, HDL, LDL, AST/ALT ratio, UA, ALP and hsCRP.

\subsection{Subgroup analysis}


Additionally, in subgroup analyses, the significant association of SAH levels with increased prevalence of NAFLD was observed in females and those with lower HOMA-IR and lower TG (all $P<0.001$ ). As for Hcy, the positive association was detected in younger participants and those with lower BMI and HOMA-IR (all $P<0.05)$. Interactions between quartiles of SAH and Hcy and stratified factors were significant in HOMAIR stratification (both $P$ for interaction $<0.05$ ). Besides, subgroup analysis also showed an inconsistently inverse association between SAM/SAH ratio and NAFLD, with significant interactions by age and HOMAIR categories (both $\mathrm{P}$ for interaction $<0.05$ ). However, there were no associations between SAM and NAFLD consistently in all subgroups analyzed. (Fig. 2)

\subsection{Correlation of methionine metabolites and degree of hepatic steatosis}

Next, we analyzed whether concentrations of serum methionine metabolites linked with the severity of hepatic steatosis which was defined semi-quantitatively and rated as absent, mild, moderate or severe based on ultrasonographic features. As shown in Fig. 3A-D, the concentrations of SAM, SAH and Hcy significantly increased with the degree of hepatic steatosis (all $P$ for trend $<0.01$ ). Conversely, SAM/SAH ratio significantly decreased as the degree of hepatic steatosis increased in all participants ( $P$ for trend < 0.001). Also, in the light of the results of ANCOVA (Fig. 3E-H), comparable associations continued to be observed in serum SAH, Hcy levels and SAM/SAH ratio (log-transformed) after adjusting for relevant confounders, including demographic characteristics, physical fitness indexes, history of diseases, lifestyle and biochemical indices (all $P$ for trend $<0.001$ ). However, serum SAM level (log-transformed) no longer increased with the degree of hepatic steatosis.

\section{Discussion}

In this observational study, we found that subjects with NAFLD had higher serum SAH and Hcy levels and lower SAM/SAH ratio than those without NAFLD in middle-aged and elderly Chinese. The results of the current study demonstrated that SAH, Hcy levels and SAM/SAH ratio were associated with NAFLD, which was consistently observed in both univariable and multivariable adjusted analyses. Furthermore, with the degree of hepatic steatosis increasing, serum SAH and Hcy concentrations tended to increase and SAM/SAH ratio tended to decrease.

Methionine metabolism is involved in a variety of physiological processes, such as the homeostasis of methionine, methylation, biosynthesis of thymidine and purine, and redox defense ${ }^{[26]}$. Due to the essential role of methionine metabolites, their disorder can result in a series of diseases, including metabolic syndrome ${ }^{[27]}$. In a cross-sectional study of 1108 Swedish individuals, an independent association between Hcy and serum insulin was closely related to metabolic syndrome ${ }^{[28]}$. NAFLD is the manifestation of metabolic syndrome in the liver. In the NAFLD population, insulin's ability to inhibit glucose and LDL production is impaired ${ }^{[29]}$. Some studies have suggested that subjects with metabolic syndrome frequently exhibited elevated ALT and lipid droplets levels and a higher prevalence of 
NAFLD ${ }^{[30]}$. In our study, participants with NAFLD had higher metabolic relevant risk factors, higher SAM, SAH, Hcy levels and lower SAM/SAH ratio than those without NAFLD. Parallelly, SAM, SAH, Hcy was positively correlated with metabolic relevant risk factors, and SAM/ SAH was inversely related with metabolic relevant risk factors. These findings hinted that methionine metabolites were associated with metabolic disorders, which may be closely linked to the development of NAFLD.

In the current circumstances, a few studies have explored the association between methionine metabolites and NAFLD, and the conclusions were still controversial. For instance, Haijiang Dai et al. reported that serum Hcy levels were positively associated with NAFLD, particularly in female, obese or non-smoking adults ${ }^{[20]}$. But some studies held contrary opinions that Hcy was independently inversely associated with NAFLD and NASH ${ }^{[21]}$. The possible reasons for these inconsistencies may be as follows. First of all, the sample size of the population was insufficient, resulting in potential biases. Secondly, these studies varied in race and disease stage. Last but not least, in these studies, methionine metabolites were often measured by high-performance liquid chromatography with fluorescence detection which was not enough sensitive. Thus, the above limitations might negatively impact the extrapolation of those conclusions. Herein, our study enrolled 2814 middle-aged and elderly community individuals in southern China and investigated a series of methionine metabolites which was detected by UHPLC/MS-MS with higher sensitivity and specificity. We found that fully adjusted ORs $(95 \% \mathrm{Cl})$ in quartile 4 of SAH, Hcy, SAM/SAH ratio for NAFLD were 1.65 (1.27-2.14), $1.63(1.26-2.12)$ and 0.63 (0.49-0.83), respectively (all $P$ for trend $<0.01$ ). Our findings suggested that methionine metabolites might be predictors and risk factors of the presence of NAFLD.

The mechanisms by which elevated serum methionine metabolites such as SAH and HCY were negatively associated with the presence of NAFLD could be as followings. It has been reported that elevated methionine metabolites could disrupt the methylation of a variety of substances, including phosphatidylcholine (PC) and sterol regulatory element-binding proteins (SREBPs). Hepatic PC is produced via SAM-dependent methylation of phosphatidylethanolamine. PC plays an essential role in maintaining hepatic lipid homeostasis by regulating lipid transport. Decreased PC level weakens the assembly and secretion of lipoproteins especially low-density lipoprotein which impair lipid excretion and further accelerate intracellular lipid droplet accumulation in the liver ${ }^{[17]}$. An epidemiological study also showed that participants with hepatic steatosis had 25\% less PC in the liver than the normal population ${ }^{[31]}$. On the other hand, SREBP-1, a transcription factor regulating lipogenesis genes in mammals, can be activated by low PC levels in a feedback mechanism. Activated SREBP-1 subsequently upregulates genes involved in lipid biosynthesis, boosting lipid droplet formation and leading to lipid accumulation in hepatocytes as well ${ }^{[16]}$. Therefore, not only impaired lipid excretion but also increased lipogenesis can promote the occurrence of NAFLD. In addition, higher SAH or Hcy could promote the progression of inflammation in the liver. Arumugam et al. reported that elevated SAH increased release of pro-inflammatory cytokines from adipocytes ${ }^{[32]}$. Cristiane et al. showed that elevated Hcy induced oxidative stress and recruited inflammatory cells in the liver of rats ${ }^{[33]}$. 
Notably, there was no association of serum SAM level with NAFLD and no linear trend between SAM and the severity of hepatic steatosis on multivariable analyses. As a dynamic reaction loop, once methionine metabolism is impaired, the reaction process will be blocked to varying degrees and substances in all links will increase. Indeed, in our study, we observed that the serum levels of SAM, SAH and Hcy in the NAFLD population were significantly higher than those without NAFLD. Higher SAM can provide more methyl donors indicating the greater methylation capacity, which is considered to be beneficial to health ${ }^{[34]}$. However, this beneficial effect may be masked by the stronger harmful effects of increased SAH and Hcy. The decreased SAM/SAH ratio suggested that the increase of SAM was much less than that of SAH in the NAFLD population. Additionally, Lind et al. reported that in population with metabolic syndrome factors, SAH or Hcy, not SAM, were related to some metabolic characteristics, such as BMI,

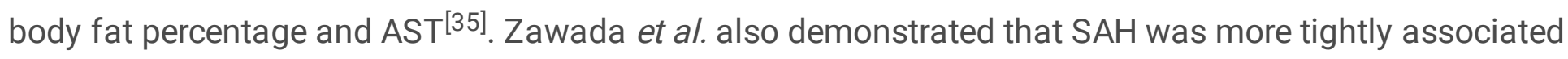
with traditional cardiovascular risk factors than SAM in a cardiovascular low-risk population ${ }^{[36]}$. Given $\mathrm{SAH}$ is the strong inhibitor of methylation, we speculate that $\mathrm{SAH}$ is a more sensitive indicator to the methylation status compared to SAM, and the decrease of SAM/SAH ratio (methylation capacity index) is mainly due to the increase of SAH levels. This conjecture needs further confirmation.

This study has several limitations. Firstly, the cross-sectional design cannot clarify the causal inference. Whether methionine metabolites are bystanders, causal factors or consequences of NAFLD cannot be answered from the results of this cross-sectional study, and prospective studies are warranted. Secondly, since all study participants had been voluntarily recruited, there is potential for selection bias. Thirdly, NAFLD was diagnosed non-invasively as appropriate for epidemiological studies generally. Nevertheless, compared to liver biopsy, ultrasonography cannot provide histological information to further explore the association between methionine metabolites and NASH. Lastly, all study participants were exclusively Chinese, which restricts the generalizability to other ethnic populations.

\section{Conclusions}

In conclusion, in this cross-sectional study of middle-aged and elderly Chinese, serum SAH and Hcy levels were positively associated with the risk of NAFLD prevalence, and SAM/SAH ratio was inversely related to NAFLD.

\section{Abbreviations}

ALP, Alkaline phosphatase; ALT, alanine aminotransferase; ANCOVA, analysis of covariate; ANOVA, analysis of variance; AST, aspartate aminotransferase; ATP, adenosine triphosphate; BMI, body mass index; $\mathrm{Cl}$, confidence interval; DBP, diastolic blood pressure; GNHS, Guangzhou Nutrition and Health Study; Hcy, homocysteine; HDL-C, high-density lipoprotein cholesterol; HOMA-IR, homoeostasis model assessment of insulin resistance; hsCRP, high-sensitivity C-reactive protein; LDL-C, low-density lipoprotein cholesterol; LOD, limit of detection; LOQ, limit of quantification; MAT, methionine adenosyltransferase; MET, metabolic equivalent of task; MS, methionine synthase; MT, methyltransferase; NAFLD, nonalcoholic fatty liver disease; NASH, non-alcoholic steatohepatitis; OR, odds ratio; SAH, S- 
adenosylhomocysteine; SAHH, S-adenosylhomocysteine hydrolase; SAM, S-adenosylmethionine; SBP, systolic blood pressure; SREBPs, sterol regulatory element-binding proteins; TC, total cholesterol; TG, triglycerides; UA, uric acid; UHPLC-MS/MS, ultra-high performance liquid chromatography-tandem mass spectrometry; WHR, waist-to-hip ratio.

\section{Declarations}

\section{Ethics approval and consent to participate}

Written informed consent was provided by all participants, and the study was approved by the Ethics Committee of the School of Public Health at Sun Yat-sen University (Approval Number ZDGWYL2009-3, Registration Number NCT03179657, www.ClinicalTrials.gov), which meets the ethical standards established in the Declaration of Helsinki.

\section{Consent for publication}

Not applicable.

\section{Availability of data and materials}

The datasets generated and/or analysed during the current study are not publicly available, since ethics approval and participants' consent does not allow public sharing of data, but are available from the corresponding author on reasonable request.

\section{Competing interest}

None.

\section{Funding}

This work was supported by the National Natural Science Foundation of China (grant No.81730090 and No.81973022).

\section{Author's contributions}

Yi Tang: Conceptualization, Data curation, Software, Formal analysis, Original Draft; Xu Chen: Methodology, Investigation, Formal analysis, Software, Original Draft; Qian Chen: Supervision, review \& editing; Jinghe Xiao: Investigation, Formal analysis, Data Curation, Software, Resources; Jiaxin Mi: Investigation, Data Curation, Software, Resources; Qiannan Liu: Data curation, Validation, Visualization; Yiran You: Validation, Visualization; Yuming Chen: Supervision, Project administration, Funding acquisition; Wenhua Ling: Conceptualization, Supervision, Funding acquisition, Review \& Editing.

\section{Acknowledgements}

We thank the staff and participants for their important contributions. 


\section{References}

[1] Younossi, Z., F. Tacke, M. Arrese, et al., Global Perspectives on Nonalcoholic Fatty Liver Disease and Nonalcoholic Steatohepatitis. Hepatology, 2019. 69(6): 2672-

2682. http://doi.org/10.1002/hep.30251

[2] Zhou, J.H., F. Zhou, W.X. Wang, et al., Epidemiological Features of Nafld from 1999 to 2018 in China. Hepatology, 2020. 71(5): 1851-1864. http://doi.org/10.1002/hep.31150

[3] Powell, E.E., V.W.S. Wong, and M. Rinella, Non-Alcoholic Fatty Liver Disease. Lancet, 2021. 397(10290): 2212-2224. http://doi.org/10.1016/S0140-6736(20)32511-3

[4] Yki-Jarvinen, H., Non-Alcoholic Fatty Liver Disease as a Cause and a Consequence of Metabolic Syndrome. Lancet Diabetes \& Endocrinology, 2014. 2(11): 901-910. http://doi.org/10.1016/S22138587(14)70032-4

[5] Reid, B.M. and A.J. Sanyal, Evaluation and Management of Non-Alcoholic Steatohepatitis. European Journal of Gastroenterology \& Hepatology, 2004. 16(11): 11171122. http://doi.org/10.1097/00042737-200411000-00005

[6] Ducker, G.S. and J.D. Rabinowitz, One-Carbon Metabolism in Health and Disease. Cell Metabolism, 2017. 25(1): 27-42. http://doi.org/10.1016/j.cmet.2016.08.009

[7] Friso, S., S. Udali, D. De Santis, et al., One-Carbon Metabolism and Epigenetics. Molecular Aspects of Medicine, 2017. 54: 28-36. http://doi.org/10.1016/j.mam.2016.11.007

[8] Xiao, Y.J., X.F. Su, W. Huang, et al., Role of S-Adenosylhomocysteine in Cardiovascular Disease and Its Potential Epigenetic Mechanism. International Journal of Biochemistry \& Cell Biology, 2015. 67: 158166. http://doi.org/10.1016/j.biocel.2015.06.015

[9] Kruglova, M.P., S.V. Grachev, P.O. Bulgakova, et al., Low S-Adenosylmethionine/SAdenosylhomocysteine Ratio in Urine Is Associated with Chronic Kidney Disease. Laboratory Medicine, 2020. 51(1): 80-85. http://doi.org/10.1093/labmed/lmz035

[10] Valli, A., J.J. Carrero, A.R. Qureshi, et al., Elevated Serum Levels of S-Adenosylhomocysteine, but Not Homocysteine, Are Associated with Cardiovascular Disease in Stage 5 Chronic Kidney Disease Patients. Clin Chim Acta, 2008. 395(1-2): 106-10. http://doi.org/10.1016/j.cca.2008.05.018

[11] Schalinske, K.L. and A.L. Smazal, Homocysteine Imbalance: A Pathological Metabolic Marker. Advances in Nutrition, 2012. 3(6): 755-762. http://doi.org/10.3945/an.112.002758

[12] Muzurovic, E., I. Kraljevic, M. Solak, et al., Homocysteine and Diabetes: Role in Macrovascular and Microvascular Complications. J Diabetes Complications, 2021. 35(3):

107834. http://doi.org/10.1016/j.jdiacomp.2020.107834 
[13] da Silva, R.P., B.J. Eudy, and R. Deminice, One-Carbon Metabolism in Fatty Liver Disease and Fibrosis: One-Carbon to Rule Them All. Journal of Nutrition, 2020. 150(5): 994-

1003. http://doi.org/10.1093/jn/nxaa032

[14] Zhang, X.L., Y. Wang, and P.S. Liu, Omic Studies Reveal the Pathogenic Lipid Droplet Proteins in Non-Alcoholic Fatty Liver Disease. Protein \& Cell, 2017. 8(1): 4-13. http://doi.org/10.1007/s13238-0160327-9

[15] Dahlhoff, C., S. Worsch, M. Sailer, et al., Methyl-Donor Supplementation in Obese Mice Prevents the Progression of Nafld, Activates Ampk and Decreases Acyl-Carnitine Levels. Molecular Metabolism, 2014. 3(5): 565-580. http://doi.org/10.1016/j.molmet.2014.04.010

[16] Walker, A.K., R.L. Jacobs, J.L. Watts, et al., A Conserved Srebp-1/Phosphatidylcholine Feedback Circuit Regulates Lipogenesis in Metazoans. Cell, 2011. 147(4): 840-

52. http://doi.org/10.1016/j.cell.2011.09.045

[17] da Silva, R.P., K.B. Kelly, A. Al Rajabi, et al., Novel Insights on Interactions between Folate and Lipid Metabolism. Biofactors, 2014. 40(3): 277-283. http://doi.org/10.1002/biof.1154

[18] Lai, Z.W., J.L. Chen, C.H. Ding, et al., Association of Hepatic Global DNA Methylation and Serum One-Carbon Metabolites with Histological Severity in Patients with Nafld. Obesity, 2020. 28(1): 197205. http://doi.org/10.1002/oby.22667

[19] Baric, I., K. Fumic, B. Glenn, et al., S-Adenosylhomocysteine Hydrolase Deficiency in a Human: A Genetic Disorder of Methionine Metabolism. Proceedings of the National Academy of Sciences of the United States of America, 2004. 101(12): 4234-4239. http://doi.org/10.1073/pnas.0400658101

[20] Dai, H.J., W.J. Wang, X.H. Tang, et al., Association between Homocysteine and Non-Alcoholic Fatty Liver Disease in Chinese Adults: A Cross-Sectional Study. Nutrition Journal, 2016.

15. http://doi.org/10.1186/s12937-016-0221-6

[21] Polyzos, S.A., J. Kountouras, K. Patsiaoura, et al., Serum Homocysteine Levels in Patients with Nonalcoholic Fatty Liver Disease. Annals of Hepatology, 2012. 11(1): 68-

76. http://doi.org/10.1016/S1665-2681(19)31488-7

[22] Fan, J.G., J.D. Jia, Y.M. Li, et al., Guidelines for the Diagnosis and Management of Nonalcoholic Fatty Liver Disease: Update 2010. Journal of Digestive Diseases, 2011. 12(1): 38-

44. http://doi.org/10.1111/j.1751-2980.2010.00476.x

[23] Arning, E. and T. Bottiglieri, Quantitation of S-Adenosylmethionine and S-Adenosylhomocysteine in Plasma Using Liquid Chromatography-Electrospray Tandem Mass Spectrometry. Methods Mol Biol, 2016. 1378: 255-62. http://doi.org/10.1007/978-1-4939-3182-8_27 
[24] Nelson, B.C., C.M. Pfeiffer, L.T. Sniegoski, et al., Development and Evaluation of an Isotope Dilution Lc/Ms Method for the Determination of Total Homocysteine in Human Plasma. Analytical Chemistry, 2003. 75(4): 775-784. http://doi.org/10.1021/ac0204799

[25] Saadeh, S., Z.M. Younossi, E.M. Remer, et al., The Utility of Radiological Imaging in Nonalcoholic Fatty Liver Disease. Gastroenterology, 2002. 123(3): 745-50. http://doi.org/10.1053/gast.2002.35354

[26] Lind, M.V., L. Lauritzen, A. Ross, et al., The Role of One Carbon Metabolism and Methylation Capacity in Metabolic Syndrome. Annals of Nutrition and Metabolism, 2015. 67: 268268. http://doi.org/10.1016/j.numecd.2018.01.005

[27] Finer, S., P. Saravanan, G. Hitman, et al., The Role of the One-Carbon Cycle in the Developmental Origins of Type 2 Diabetes and Obesity. Diabetic Medicine, 2014. 31(3): 263-

272. http://doi.org/10.1111/dme.12390

[28] Bjorck, J., M. Hellgren, L. Rastam, et al., Associations between Serum Insulin and Homocysteine in a Swedish Population-a Potential Link between the Metabolic Syndrome and Hyperhomocysteinemia: The Skaraborg Project. Metabolism, 2006. 55(8): 1007-13. http://doi.org/10.1016/j.metabol.2006.03.010

[29] Muzurovic, E., D.P. Mikhailidis, and C. Mantzoros, Non-Alcoholic Fatty Liver Disease, Insulin Resistance, Metabolic Syndrome and Their Association with Vascular Risk. Metabolism-Clinical and Experimental, 2021. 119. http://doi.org/10.1016/j.metabol.2021.154770

[30] Seppala-Lindroos, A., S. Vehkavaara, A.M. Hakkinen, et al., Fat Accumulation in the Liver Is Associated with Defects in Insulin Suppression of Glucose Production and Serum Free Fatty Acids Independent of Obesity in Normal Men. Journal of Clinical Endocrinology \& Metabolism, 2002. 87(7): 3023-3028. http://doi.org/10.1210/jcem.87.7.8638

[31] Puri, P., R.A. Baillie, M. Wiest, et al., A Lipidomic Analysis of Non-Alcoholic Fatty Liver Disease (Nafld). Journal of Hepatology, 2006. 44: S260-S261. http://doi.org/10.1016/S0168-8278(06)80708-7

[32] Arumugam, M.K., S. Chava, K. Rasineni, et al., Elevated S-Adenosylhomocysteine Induces Adipocyte Dysfunction to Promote Alcohol-Associated Liver Steatosis. Scientific Reports, 2021. 11(1). http://doi.org/10.1038/s41598-021-94180-x

[33] Matte, C., F.M. Stefanello, V. Mackedanz, et al., Homocysteine Induces Oxidative Stress, Inflammatory Infiltration, Fibrosis and Reduces Glycogen/Glycoprotein Content in Liver of Rats. International Journal of Developmental Neuroscience, 2009. 27(4): 337-

344. http://doi.org/10.1016/j.ijdevneu.2009.03.005

[34] Pfalzer, A.C., S.W. Choi, S.A. Tammen, et al., S-Adenosylmethionine Mediates Inhibition of Inflammatory Response and Changes in DNA Methylation in Human Macrophages. Physiological Genomics, 2014. 46(17): 617-623. http://doi.org/10.1152/physiolgenomics.00056.2014 
[35] Lind, M.V., L. Lauritzen, H. Vestergaard, et al., One-Carbon Metabolism Markers Are Associated with Cardiometabolic Risk Factors. Nutr Metab Cardiovasc Dis, 2018. 28(4): 402-

410. http://doi.org/10.1016/j.numecd.2018.01.005

[36] Zawada, A.M., K.S. Rogacev, B. Hummel, et al., S-Adenosylhomocysteine Is Associated with Subclinical Atherosclerosis and Renal Function in a Cardiovascular Low-Risk Population. Atherosclerosis, 2014. 234(1): 17-22. http://doi.org/10.1016/j.atherosclerosis.2014.02.002

\section{Figures}

\section{Guangzhou Nutrition and Health Study (GNHS)}

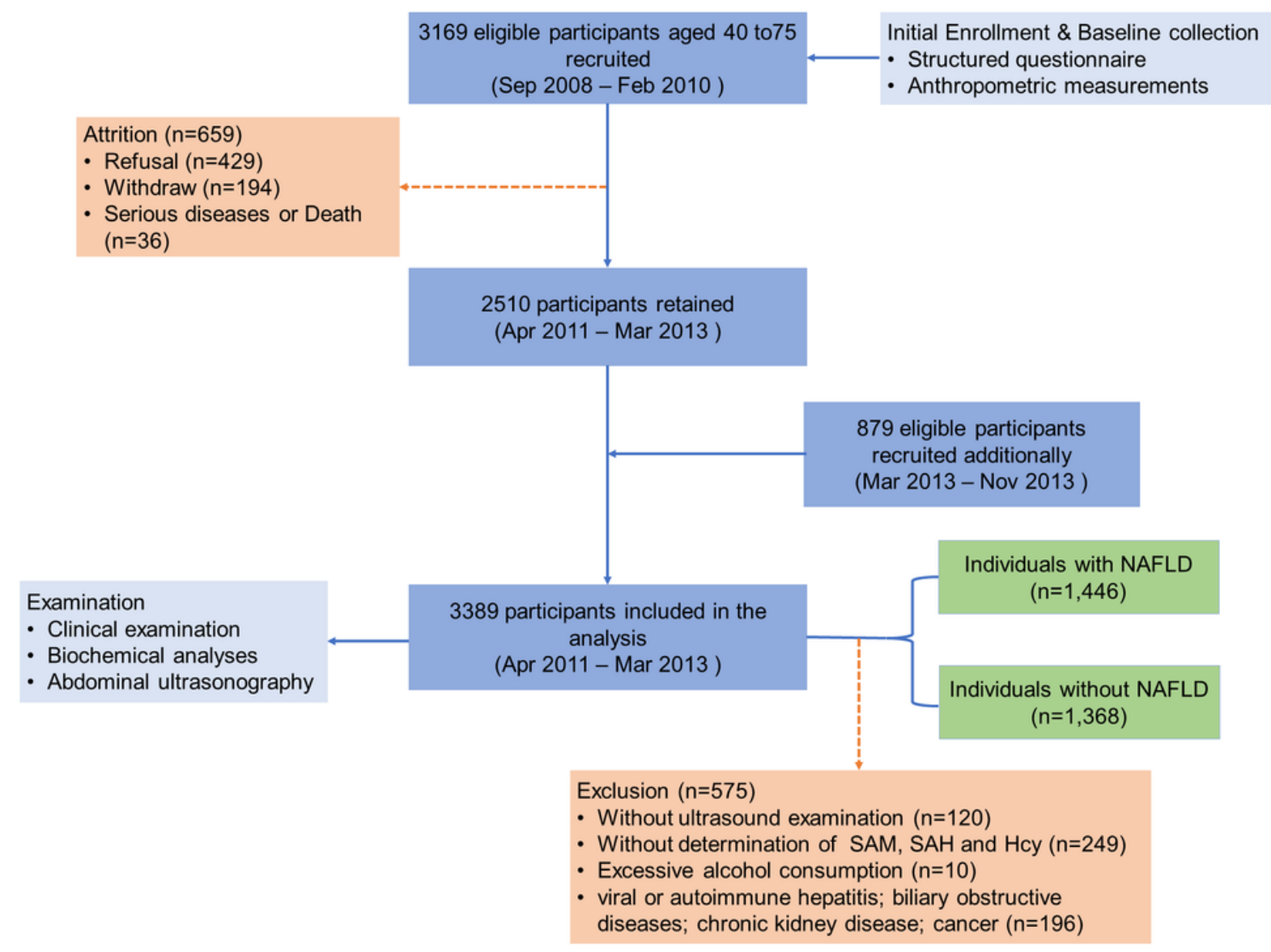

Figure 1

Overview of the study population. NAFLD, non-alcoholic fatty liver disease; SAM, S-adenosylmethionine; SAH, S-adenosylhomocysteine; Hcy, homocysteine. 
Adjusted ORs $(95 \% \mathrm{Cl})$ of serum SAM levels for NAFLD

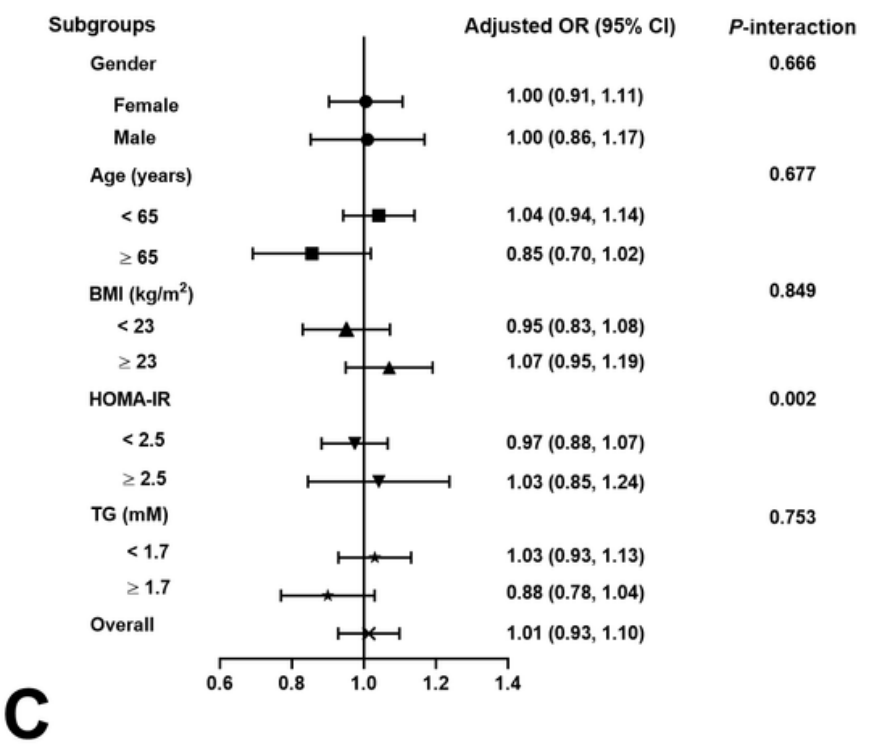

Adjusted ORs $(95 \% \mathrm{Cl})$ of serum Hcy levels for NAFLD

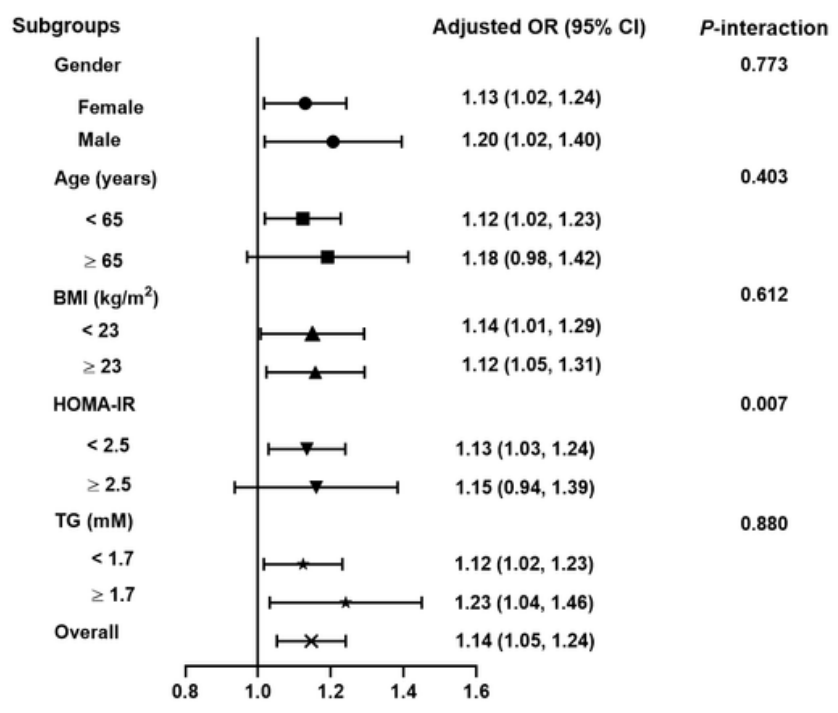

Adjusted ORs $(95 \% \mathrm{CI})$ of serum SAH levels for NAFLD

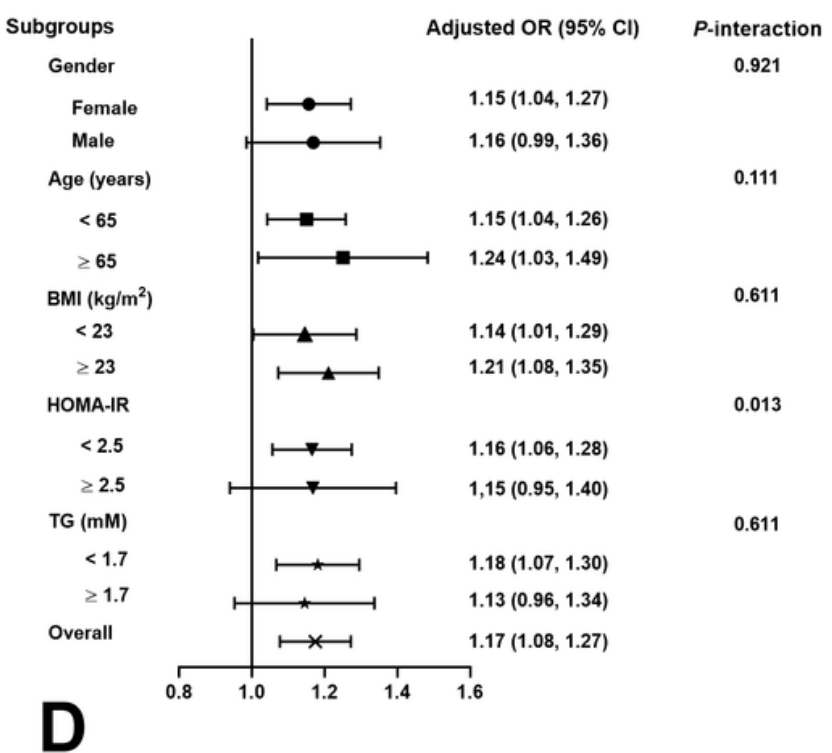

Adjusted ORs $(95 \% \mathrm{CI})$ of serum SAM/SAH ratio for NAFLD

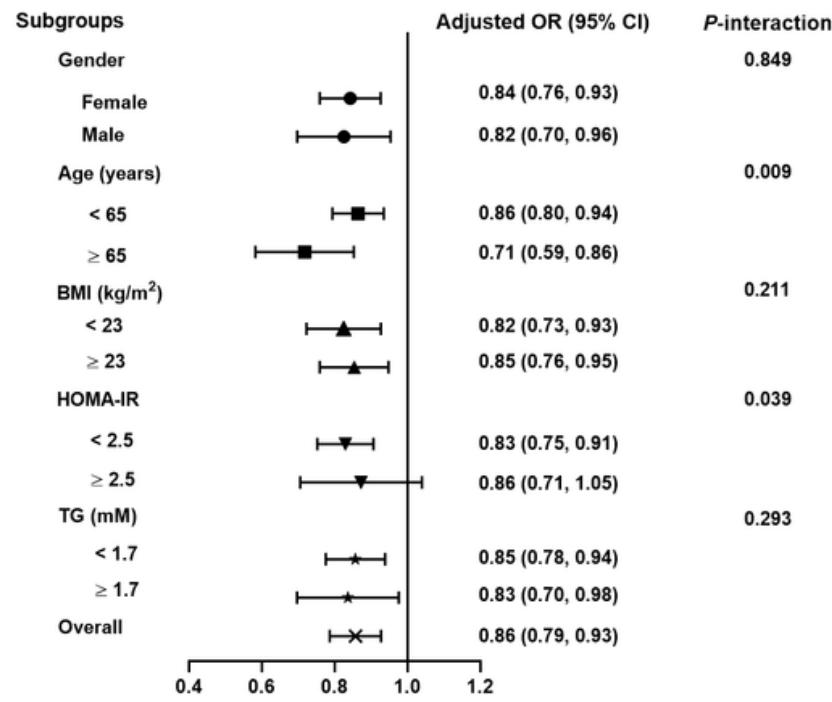

Figure 2

Subgroup analysis for by gender, age, BMI, HOMA-IR and TG using multivariable logistic regression. The data are shown as the ORs $(95 \% \mathrm{Cl})$ in each quartile of serum (A) Hcy, (B) SAH, (C) SAM, (D) SAM/SAH levels for NAFLD. 
A

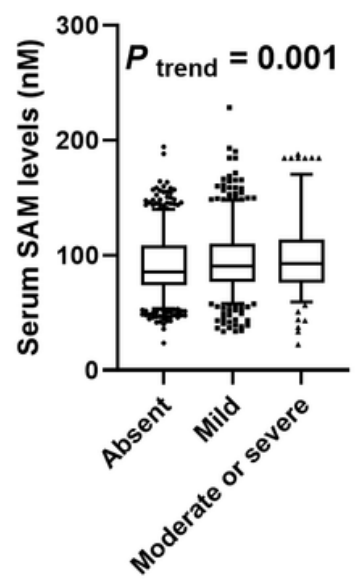

$E$

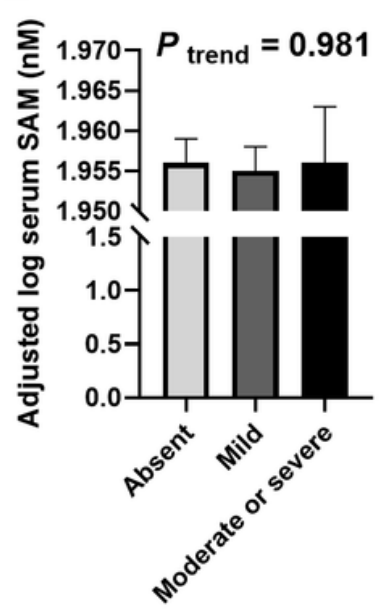

B

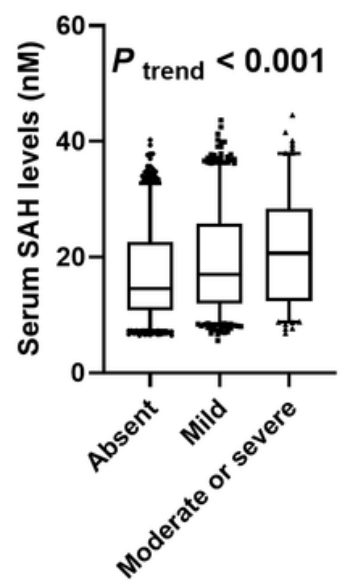

$\mathbf{F}$

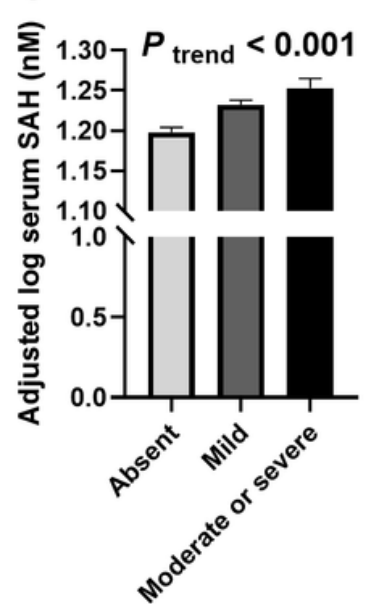

C

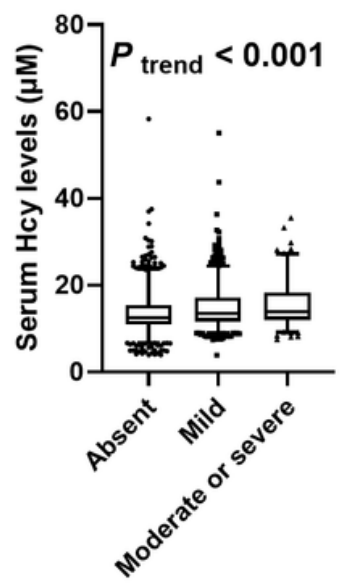

G

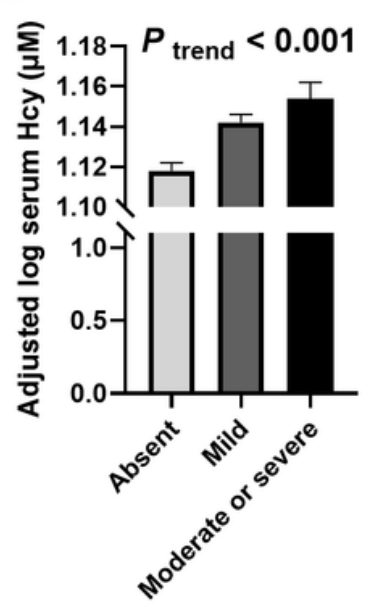

D
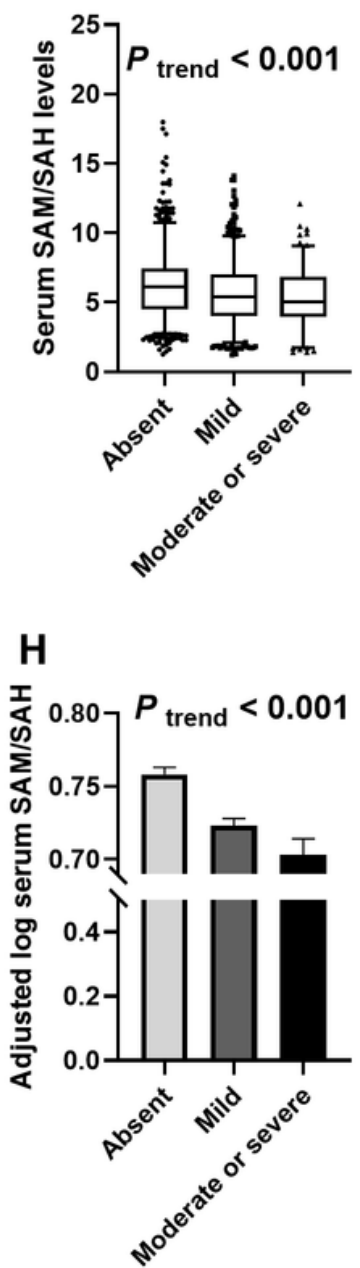

Figure 3

Correlation of methionine metabolites and degree of hepatic steatosis. (A-D) univariate and (E-H) multivariate. $A$, univariate $P$ values are calculated by were analyzed by Kruskal-Wallis one-way ANOVA for k samples. B, serum (E) SAM, (F) SAH, (G) Hcy, (H) SAH/SAM ratio (log-transformed) were adjusted for age, gender, BMI, WHR, trunk fat ratio, physical activity, current smoking, current drinking, history of hypertension, diabetes, dyslipidemia and heart disease, HOMA-IR, AST/ALT ratio, TC, TG, HDL, LDL, UA, ALP and hsCRP. Multivariate P values are analyzed by ANCOVA.

\section{Supplementary Files}

This is a list of supplementary files associated with this preprint. Click to download.

- FigS1NM.tif

- TableS1.docx 